\title{
L'orientation des vols d'Adonia variegata Goeze, Coccinella septempunctata L. et Propylea quatuordecimpunctata L. (Col., coccinellidae) vers un champ de blé au printemps
}

\author{
A. Ferran, G. Iperti, L. Lapchin et J.M. Rabasse \\ INRA, station de zoologie et de lutte biologique, 37, boulevard du Cap, 06602 Antibes, France \\ (reçu le 10 décembre 1988; accepté le 12 juin 1989)
}

Résumé - Des pièges englués ont été disposés autour d'un champ de blé pour étudier les modalités de sa colonisation par les coccinelles au printemps.

Dans cette agrobiocénose particulière, les adultes d'Adonia variegata, de Coccinella septempunctata et de Propylea quatuordecimpunctata arrivent globalement suivant le sens nord-sud et plus précisément selon 2 directions, du nord-est vers le sud-ouest et du nord-ouest vers le sud-est. Ces vols de colonisation se font contre le vent dominant et sont de faible altitude.

Ces observations sont discutées en fonction de certaines caractéristiques biotiques du champ de blé (densité du végétal et des proies, distribution des coccinelles), de son environnement (champ de luzerne, haie de cyprès, colline) et des résultats bibliographiques antérieurs.

piège adhésif - vol - colonisation - blé - environnement

Summary - Flight orientation of Adonia variegata, Coccinella septempunctata and Propylea quatuordecimpunctata toward a field in spring time. Sticky traps were set around a corn field in order to study how it is colonized by coccinellids during Spring time.

In this peculiar agrobiocoenose, adults of Adonia variegata, Coccinella septempunctata and Propylea quatuordecimpunctata arrived generally from a southward direction and, to be more precise, from 2 main directions; south-westward and south-eastward. These colonizing flights are upwind and of low altitude.

The results of this study are discussed in relation to some biotic features of cornfield (crop and aphid densities, coccinellid distribution), to its environment (hill, alfalfa field, cypress hedge) and to other works on this topic.

adhesive trap - flight - colonisation - wheat - environment

\section{INTRODUCTION}

Lorsque les conditions climatiques sont favorables, la présence de coccinelles aphidiphages dans un champ dépend de facteurs liés à la fois à cette culture et à l'environnement.

Lors de leurs déplacements, après l'hivernation, ou durant le printemps pour les espèces polyvoltines, ces prédateurs recherchent une strate végétale préférentielle (Iperti, 1965) une certaine densité du végétal (Honek, 1979) et des proies (Ives, 1981), plus généralement, des conditions microclimatiques particulières, souvent propres à l'espèce (Ewert \& Chiang, 1966).

La localisation géographique, par rapport à la culture, considérée des sites d'estivo-hivernation ou de foyers précoces de pucerons sur des plantes spontanées (Stechmann, 1984) ou cultivées (Ives, 1981) sont autant de paramètres qui peuvent influer sur les modalités des vols de colonisation et sur la distribution initiale de ces auxiliaires dans les champs.

Ces échanges avec l'environnement sont difficiles à quantifier. Deux techniques sont générale- 
ment utilisées : le lâcher d'individus marqués (Ali \& Azam, 1977) mais le taux de recapture est faible (Kieckhefer \& Olson, 1974) ou le piégeage à l'aide de panneaux englués (Dean, 1982, Dowel \& Cherry, 1981) sur lesquels les récoltes sont souvent peu importantes (Ives, 1981).

Toutefois, de tels pièges, disposés à la périphérie d'un champ, peuvent indiquer si l'effet global de la culture et de l'environnement se traduit (ou non) par une certaine orientation des vols de colonisation. Le travail ci-dessous a été réalisé dans cette optique : des panneaux englués ont été disposés autour d'un champ de blé pour intercepter les 3 principales coccinelles $A$. variegata, $C$. septempunctata et $P$. quatuordecimpunctata.

\section{MATÉRIEL ET MÉTHODES}

Le champ de blé $\left(4125 \mathrm{~m}^{2}\right)$ est situé dans la vallée du Var (Alpes-Maritimes) (Fig. 1). Il est divisé en 4 secteurs $A, B, C, D$, de surface sensiblement égale, euxmêmes subdivisés en parcelles élémentaires de $25 \mathrm{~m}^{2}$ $(5 \times 5 \mathrm{~m})$. Son environnement immédiat est constitué, en particulier, par une colline boisée qui le domine à l'ouest et par une haie de cyprès qui le sépare au nord, nord-est d'un champ de luzerne.

Les panneaux englués sont d'un modèle comparable à ceux utilisés par Ives (1981). Ils sont constitués par une armature en bois de couleur verte $(\mathrm{H}$. : $2 \mathrm{~m}$, $1: 1,5 \mathrm{~m})$ portant une toile plastique transparente (maille : $3 \mathrm{~mm}$ ). Ces panneaux sont disposés verticalement sur le sol à $1 \mathrm{~m}$ des bordures du champ. Le côté orienté vers l'extérieur de la culture est badigeon-

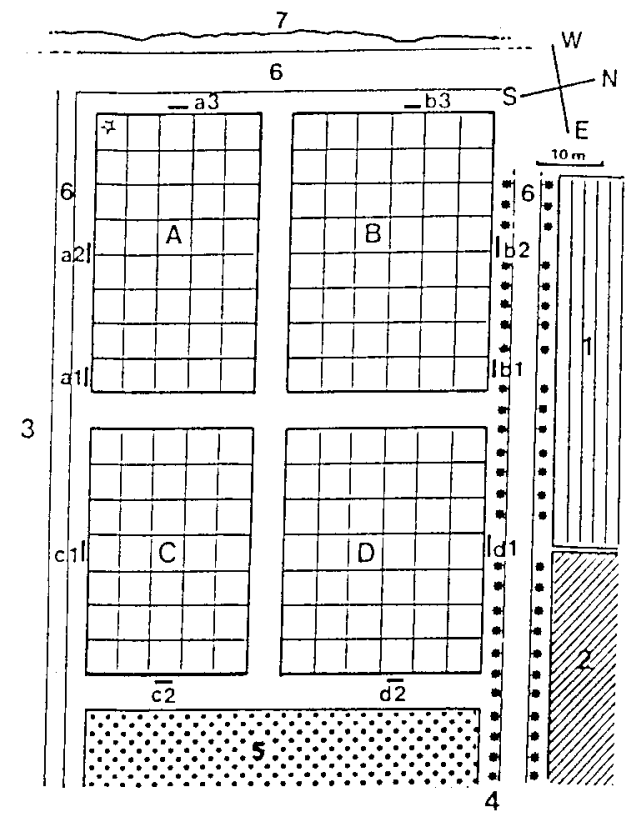

Fig. 1. Le champ de blé et son environnement immédiat : L : surface unitaire d'échantillonnage $\left(25 \mathrm{~m}^{2}\right), 1$. Pépinière de cyprès, 2. champ de luzerne $\left(2500 \mathrm{~m}^{2}\right), 3$. terrain vague, 4. haie de cyprès, 5 . champ de maïs $\left(2000 \mathrm{~m}^{2}\right), 6$. route et chemins, 7 . forêt de pins. né avec une glue non insecticide (Tanglefoot). Selon leur position autour du champ, ils ont été appelés $a_{1}$, $a_{2}, a_{3}, b_{1}, b_{2}, b_{3}, c_{1}, c_{2}, d_{1}$ et $d_{2}$ (Fig. 1). En 1987, chacun d'eux a été divisé en 3 bandes horizontales de même surface (haut, moyen, bas).

Les adultes capturés par ces pièges sont récoltés chaque semaine et sont disséqués en laboratoire (détermination du sexe, état des ovaires).

Dans le champ de blé, l'état de la végétation (nombre de talles par $\mathrm{m}^{2}$, hauteur en $\mathrm{cm}$ ), l'importance numérique des populations aphidiennes (nombre par $\mathrm{m}^{2}$ ) et des coccinelles (nombre total par secteur) sont estimés à l'aide des méthodes proposées par Lapchin et al. (1987). Dans 20 parcelles unitaires $\left(25 \mathrm{~m}^{2}, 5\right.$ par secteur) sont déterminés le nombre de raies, le nombre de pieds et de talles portés par 3 fois $0,50 \mathrm{~cm}$ de rang. Trois pieds de blé, prélevés sur chacune de ces longueurs, sont ramenés au laboratoire pour estimer la hauteur des talles et le nombre de pucerons présents. Les coccinelles adultes sont dénombrées dans toutes les parcelles unitaires par un comptage visuel rapide ( 2 min par $\mathrm{m}^{2}$ ).

Les populations de ces coccinelles ont été contrôlées de 1984 à 1987 alors que les piégeages et la détermination des conditions biotiques (croissance du blé, densité des proies) n'ont été effectués qu'en 1986 et 1987.

\section{RÉSULTATS}

\section{Principales caractéristiques biotiques des différents secteurs}

En raison de l'hétérogénéité du sol, la couverture végétale du champ n'est pas exactement la même dans les différents secteurs (Tableau I a et b). En 1987 en particulier, le secteur A, avec un nombre de talles sensiblement plus élevé et une hauteur moyenne des talles nettement supérieure, présente la plus grande densité de végétation et un sol entièrement couvert; le secteur $D$ est à l'opposé du précédent; les secteurs B, C, sont intermédiaires.

L'importance des populations aphidiennes à l'arrivée des premiers adultes de $C$. septempunctata est différente d'une année à l'autre : elle est maximale en D en 1986, en A en 1987. Lors du maximum de population de cette coccinelle (en 1986 et 1987), les pucerons sont moins nombreux en $A$ et atteignent des niveaux de population de près du double dans les 3 autres secteurs.

\section{La distribution des coccinelles dans le champ (Tableau II)}

Quelle que soit l'année, le nombre total d'adultes de $C$. septempunctata issus des sites d'hivernation est plus élevé dans les secteurs $(B+D)$ que dans les secteurs $(A+C)$. 
Tableau I a. - 1986 - Principales caractéristiques biotiques des secteurs A, B, C, D du champ de blé (Résultats : moyenne d'échantillons prélevés dans des parcelles de $25 \mathrm{~m}^{2}$ ).

Critères biologiques

Secteurs du champ de blé

$\begin{array}{llll}A & B & C & D\end{array}$

Année 1986 :- Arrivée des adultes de C. septempunctata (15 avril)

$\begin{array}{lcccc}\text { Nombre moyen de talles } / \mathrm{m}^{2} & 528 & 524 & 563 & 544 \\ \text { Hauteur moyenne d'une talle }(\mathrm{cm}) & 48 & 44 & 47 & 43 \\ \text { Nombre moyen d'aphides } / \mathrm{m}^{2} & 18,5 & 34,3 & 18,2 & 110,0\end{array}$

- maximum de population (13 mai)

Nombre moyen de talles $/ \mathrm{m}^{2}$

Hauteur moyenne d'une talle $(\mathrm{cm})$

Nombre moyen d'aphides $/ \mathrm{m}^{2}$

$\begin{array}{cc}494 & 456 \\ 71 & 64 \\ 159,1 & 281,9\end{array}$

449
72
365,4

261,3

Tableau I b. - 1987 - Principales caractéristiques biotiques des secteurs A, B, C, D du champ de blé (Résultats : moyenne d'échantillons prélevés dans des parcelles de $25 \mathrm{~m}^{2}$.

Critères biologiques

Secteurs du champ de blé
A
B
C
$D$

Année 1987 : - Arrivée des adultes de C. septempunctata (14 avril)

\begin{tabular}{|c|c|c|c|c|}
\hline Nombre moyen de talles $/ \mathrm{m}^{2}$ & 565 & 391 & 307 & 244 \\
\hline Hauteur moyenne d'une talle $(\mathrm{cm})$ & 42 & 31 & 18 & 22 \\
\hline Nombre moyen d'aphides $/ \mathrm{m}^{2}$ & 70,3 & 19,3 & 40,8 & 14,3 \\
\hline \multicolumn{5}{|c|}{ - maximum de population (26 mai) } \\
\hline Nombre moyen de talles $/ \mathrm{m}^{2}$ & 405 & 289 & 270 & 195 \\
\hline Hauteur moyenne d'une talle $(\mathrm{cm})$ & 67 & 31 & 18 & 22 \\
\hline Nombre moyen d'aphides $/ \mathrm{m}^{2}$ & 22,2 & 41,5 & 46,1 & 59,7 \\
\hline
\end{tabular}

A. variegata est une espèce polyvoltine qui apparaît plus tard (fin mai) dans le champ de blé. En 1984 et 1986, contrairement à 1985 et 1987, les adultes ont été plus nombreux dans les secteurs B et D.

$P$. quatuordecimpunctata est également une espèce polyvoltine et tardive qui fréquente préférentiellement la strate des arbustes (Iperti, 1965). Au cours de ces 4 années, elle a fréquenté surtout les secteurs $A$ et $C$.

Au cours de la période de végétation du blé, la population des coccinelles est constituée, en 1986 et 1987 respectivement, par $34 \%$ et $35 \%$ de C. septempunctata, $61 \%$ et $62 \%$ d'A. variegata et par $5 \%$ et $3 \%$ de $P$. quatuordecimpunctata.

\section{Les captures par les panneaux englués (Fig. 2).}

\section{Résultats toutes espèces confondues}

Pour le nombre total de captures, les panneaux englués peuvent être classés dans l'ordre d'efficacité décroissante suivant en $1987: b_{2}, d_{2}, b_{1}$, $b_{3}, d_{1}, c_{1}, c_{2}, a_{1}, a_{2}, a_{3}$, l'importance des captures sur ces 5 derniers pièges ayant varié d'une année à l'autre.

Les panneaux englués situés sur la bordure nord du champ de blé sont les plus riches en coccinelles. La colonisation semble donc s'effectuer par des vols globalement orientés du nord vers le sud, dans le sens descendant de la val- 
Tableau II. Nombre total $\left({ }^{\star}\right)$ et pourcentage $\left({ }^{\star \star}\right)$ d'adultes recensés dans les secteurs $(A+C)$ et $(B+D)$ du champ de blé, de l'arrivée jusqu'au maximum de population de chaque espèce.

Secteurs du champ

1984
Années

1985
1987

C. septempunctata

$\begin{array}{lcccr}\text { A et C } & 14^{\star \star} & 25 & 23 & 21 \\ & (102)^{*} & (259) & (100) & (129) \\ \text { B et D } & 86 & 75 & 77 & 80 \\ & (636) & (781) & (337) & (516)\end{array}$

A. variegata

A et $\mathrm{C}$

$B$ et $D$

P. quatuordecimpunctata

$\begin{array}{ccccc}\text { A et } \mathrm{C} & 67 & 72 & 69 & 70 \\ & (64) & (149) & (46) & (37) \\ \text { B et D } & 33 & 28 & 31 & 30 \\ & (32) & (53) & (21) & (16)\end{array}$

lée. Dans cette orientation générale, 2 directions particulières paraissent se dégager; elles correspondent respectivement aux panneaux $b_{2}$ et $d_{2}$.

\section{Analyse par espèce}

A. variegata est l'espèce la plus fréquente sur les panneaux englués (45 et $49 \%$ du nombre total des captures en 1986 et 1987), notamment en $b_{2}$ et $d_{2}$. Ceux-ci sont également les plus riches en C. septempunctata qui, dans l'ensemble sont moins nombreuses (1986 : 23\% - $1987: 26 \%$ ). $P$. quatuordecimpunctata est surtout abondante
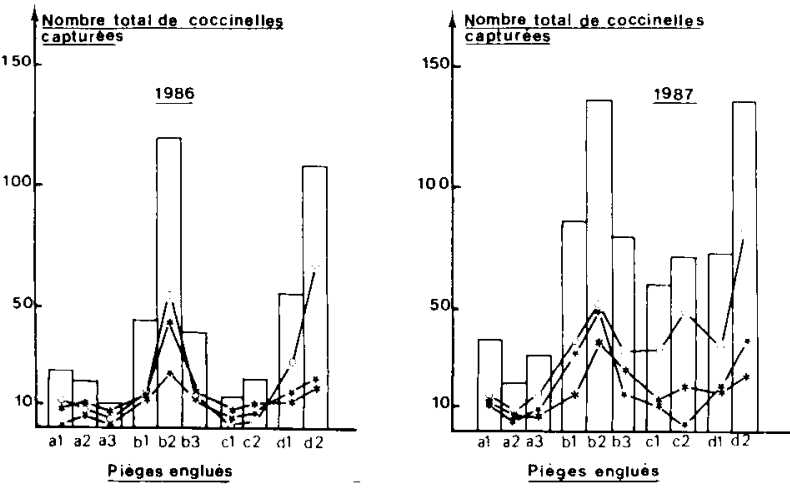

Fig. 2. Variation du nombre des captures en fonction des pièges englués (histogramme : toutes les coccinelles; $O: A$. variegata, $\star:$ C. septempunctata, $*$ : $P$. quatuordecimpunctata). sur le panneau $b_{2}$, elle représente respectivement $32 \%(1986)$ et $25 \%$ (1987) des captures totales.

La répartition des adultes de $C$. septempunctata et d' $A$. variegata sur les 10 panneaux englués est différente d'une année à l'autre $\left(\chi^{2}=\right.$ $\left.18,1, \chi^{2}=59,4\right)$. Cette variation est imputable à l'ensemble des panneaux englués a et $c$ qui ont présenté un taux de captures plus élevé en 1987. En revanche la distribution de $P$. quatuordecimpunctata est restée identique $\left(\chi^{2}=10,4\right)$.

\section{Rapport captures - distribution dans le champ}

Bien qu'il soit difficile dans cette expérimentation de rapprocher le nombre de coccinelles capturées par les différents pièges de leur distribution dans le champ, il semble que l'on puisse associer :

- pour C. septempunctata, le maximum de captures sur les panneaux $b_{1}, b_{2}$ et $d_{2}$ au plus grand pourcentage d'adultes dans les secteurs $B$ et $D$,

- pour A. variegata, l'augmentation en 1987 (par rapport à 1986) des captures par les panneaux $c$ à la variation d'une année à l'autre du pourcentage d'adultes trouvés dans les secteurs $A$ et $C$,

- pour $P$. quatuordecimpunctata, l'opposition géographique entre les panneaux où cette espè- 
ce a été capturée en plus grand nombre $\left(b_{2}, d_{2}\right.$ et $b_{3}$ en 1987) et les secteurs les plus riches en adultes ( $A$ et $C$ ).

\section{L'évolution des captures en fonction du temps}

Bien que les captures hebdomadaires soient faibles, il existe pour chaque espèce des relations linéaires significatives entre le nombre total (NC) d'adultes colonisateurs dénombrés dans le champ à chaque date d'échantillonnage et le nombre total correspondant d'individus $(N p)$ collectés sur les panneaux englués. Les différentes droites de régression ont été calculées (Tableau III).

Les adultes de $C$. septempunctata et d'A. variegata nés dans le champ, qui ont une coloration élytrale orangée, n'ont pas été pris en considération dans ces calculs. Toutefois des individus âgés sont encore capturés, mais en moins grand nombre, après le vol de colonisation. Ils témoignent ainsi de l'existence d'échanges permanents entre le champ de blé et son environnement (Ives, 1981).

Le nombre total d'adultes de $P$. quatuordecimpunctata récoltés sur les pièges est voisin ou même légèrement supérieur à celui des individus dénombrés dans l'ensemble du champ. II paraît probable que la méthode générale d'échantillonnage des coccinelles n'est pas adaptée à cette espèce en raison de sa petite taille, de sa couleur jaune voisine de celle des feuilles de blé desséchées et de sa propension à se laisser tomber à l'approche des observateurs.

\section{La distribution des coccinelles sur les pan- neaux englués (Tableaux IV et $V$ ).}

L'analyse de la distribution des coccinelles sur les 3 niveaux horizontaux (haut, moyen, bas) montre que, pour les 3 espèces, le nombre d'individus capturés croît significativement $(F=11,1$, seuil de signification $1 \%$ ) vers le bas des panneaux. II semble, par conséquent, que ces pièges interceptent essentiellement des adultes, qui effectuent des vols rasants de type alimentaire (Kennedy, 1961).

Quelle que soit l'espèce considérée, les deux sexes sont capturés dans la même proportion $(F=5,40$ ) (Tableau V).

Tableau IV. Répartition des adultes sur les pièges englués en fonction de leur hauteur ( ${ }^{*}$ nombre total, ** pourcentage. C.s. : C. septempunctata, A.v. : A. variegata, P.q. : P. quatuordecimpunctata).

\begin{tabular}{|c|c|c|c|}
\hline $\begin{array}{l}\text { Niveaux sur les } \\
\text { panneaux englués }\end{array}$ & C.s. & $\begin{array}{l}\text { ccinelle } \\
\text { A.v. }\end{array}$ & P.q. \\
\hline $\begin{array}{l}\text { Haut } \\
(133,4 \mathrm{~cm} \text { à } 200 \mathrm{~cm})\end{array}$ & $\begin{array}{l}21^{\star *} \\
(40)^{\star}\end{array}$ & $\begin{array}{c}10 \\
(37)\end{array}$ & $\begin{array}{c}18 \\
(33)\end{array}$ \\
\hline $\begin{array}{l}\text { Moyen } \\
(66,7 \mathrm{~cm} \text { à } 133,4 \mathrm{~cm})\end{array}$ & $\begin{array}{l}36 \\
(69)\end{array}$ & $\begin{array}{l}35 \\
(127)\end{array}$ & $\begin{array}{c}29 \\
(53)\end{array}$ \\
\hline $\begin{array}{l}\text { Bas } \\
\text { (0 à } 66,7 \mathrm{~cm} \text { ) }\end{array}$ & $\begin{array}{c}43 \\
(81)\end{array}$ & $\begin{array}{l}55 \\
(197)\end{array}$ & $\begin{array}{c}53 \\
(96)\end{array}$ \\
\hline
\end{tabular}

Tableau III. Relations linéaires entre le nombre $(N p)$ d'adultes capturés sur les panneaux englués chaque semaine et le nombre correspondant $(\mathrm{Nc})$ d'adultes recensés dans le champ de blé. $-n$ : nombre d'échantillons, liaison significative à $P<0,01\left(^{* \star}\right)$ ou $P<0,001\left(^{\star \star *}\right)$.

$\begin{array}{lll}\text { Espèces de } & \text { Années } & 1987 \\ \text { coccinelles } & 1986 & \end{array}$

C. septempunctata

A. variegata

P. quatuordecimpunctata

$$
\begin{aligned}
N c & =10.2 N p-24,5 \\
n & =5, r=0,96 \\
t & =5,9^{\star \star}
\end{aligned}
$$

$$
\begin{aligned}
N c & =1,8 \quad N p-6,9 \\
n & =13, r=0,88 \\
t & =6,7^{\star \star}
\end{aligned}
$$

$$
\begin{aligned}
N c & =1,1 \quad N p-9,2 \\
n & =10, r=0,79 \\
t & =3,7^{\star *}
\end{aligned}
$$

$$
\begin{aligned}
N_{c} & =19,1 \quad N p-86,6 \\
n & =8, r=0,96 \\
t & =7,7^{\star \star \star}
\end{aligned}
$$

$$
\begin{aligned}
N c & =1,7 \quad N p-2,0 \\
n & =11, r=0,84 \\
t & =4,5^{\star \star} \\
N c & =0,2 \quad N p+2,1 \\
n & =12, r=0,88 \\
t & =5,7^{\star \star \star}
\end{aligned}
$$


Tableau V. Nombre ( $\left(^{\star}\right)$ et pourcentage $\left(^{\star \star}\right)$ de coccinelles mâles et femelles capturées sur l'ensemble pièges englués (C.s. : C. septempunctata, A.v. : A. variegata, P.q. : P. quatuordecimpunctata).

\begin{tabular}{|c|c|c|c|}
\hline Sexe & C.s. & $\begin{array}{c}\text { Coccinelles } \\
\text { A.v. }\end{array}$ & P.q. \\
\hline Femelles & $\begin{array}{l}46^{* *} \\
(87)^{*}\end{array}$ & $\begin{array}{l}43 \\
(155)\end{array}$ & $\begin{array}{l}54 \\
(99)\end{array}$ \\
\hline Mâles & $\begin{array}{l}54 \\
(103)\end{array}$ & $\begin{array}{l}57 \\
(206)\end{array}$ & $\begin{array}{l}46 \\
(83)\end{array}$ \\
\hline
\end{tabular}

\section{DISCUSSION}

La colonisation d'un champ de blé par les coccinelles $A$. variegata, $C$. septempunctata et $P$. quatuordecimpunctata a été étudiée à l'aide de pièges englués disposés à la périphérie de la culture et d'un dénombrement de la population en place. Ces pièges, bien qu'ils soient recommandés par Dean (1982), présentent un certain nombre d'inconvénients : un faible taux de captures (Heathcote, 1978) qui limite la portée des analyses, des effets (attractivité, répulsivité) souvent mal appréciés sur l'orientation de ces prédateurs (Caprina \& Walmsley, 1978) et une hauteur prospectée peu importante par rapport à l'altitude que peuvent atteindre ces insectes (Ewert \& Chiang, 1966; Duelli, 1980).

Toutefois dans les conditions du champ de blé expérimental et de son environnement particulier, nous avons montré que l'immigration de ces 3 coccinelles se fait globalement du nord vers le sud, plus précisément du NE vers le SO et du NO vers le SO.

Ces vols ne sont possibles qu'au-dessus d'un certain seuil thermique (Ives, 1981). En région méditerranéenne, cette période d'activité se situe au moment de la journée où le vent dominant souffle du sud vers le nord (brise marine). Ces coccinelles envahissent le champ de blé par des vols contre le vent qui rappellent les appetitive upwind flights mis en évidence par Duelli (1980) chez Ch. carnea et qui correspondent, chez cette espèce, à la recherche d'aliments après l'atterrissage dans la culture. Au contraire, en juin, lors de l'envol vers les sites d'estivo-hivernation (migration), les adultes de $C$. septempunctata nés dans le champ, se déplacent dans le sens du vent (Iperti \& Migeon, comm. pers.) comme Ali \& Azam (1977) l'ont également constaté.

La distribution des adultes sur les pièges englués semble indiquer que ces vols de coloni- sation sont plutôt rasants. En effet, quelle que soit l'espèce, $80 \%$ des individus sont capturés à moins de $1,5 \mathrm{~m}$ du sol. D'après Davis \& Kirkland (1982), lors de leur dispersion à partir des sites d'estivo-hivernation, les coccinelles présenteraient jusqu'à la fin de la maturation sexuelle, une alternance de vols et de périodes trophiques. Ce phénomène paraît exister dans notre agrobiocénose puisque la majorité (85\%) des femelles capturées à la lisière du blé sont prêtes à pondre. Chez les 3 coccinelles considérées, comme chez la chrysope précédemment citée, la faible altitude de ces vols pourrait être liée à leur plus grande lourdeur (par rapport aux individus immatures) et à la recherche de sites d'oviposition (Kokubu \& Duelli, 1984).

L'orientation de ces vols dépend à la fois de la culture et son environnement. Nous avons pu faire un certain nombre de rapprochements entre la distribution des coccinelles dans le champ de blé et l'efficacité des différents pièges. $C$. septempunctata et $P$. quatuordecimpunctata viennent du nord et arrivent dans une zone de blé peu dense. La première espèce trouve là les conditions thermiques correspondant à ses besoins (Honek, 1979) et s'y installe tandis que la seconde doit encore traverser le champ pour trouver ses zones préférentielles à forte densité de végétal (Honek, 1979).

Dans nos conditions expérimentales il est difficile d'affirmer, contrairement à Ives (1981), que l'efficacité des pièges est fonction de la densité des pucerons dans la parcelle. L'idée, selon laquelle $C$. septempunctata serait capable de percevoir les pucerons au cours des vols et de s'orienter vers les parties des champs les plus infestées (Honek, 1980), est en contradiction avec les recherches en laboratoire qui tendent à prouver que la détection des proies se fait uniquement lors du contact physique (Hodek, 1973; Nakamuta \& Saito, 1985).

L'environnement ou plus particulièrement la composition des agrobiocénoses a une grande influence sur la densité des coccinelles (Andow \& Risch, 1985). Dans notre étude, l'arrivée des coccinelles, en particulier de $C$. septempunctata, selon la direction NE-SO est très probablement en rapport avec la présence de la parcelle de luzerne située au nord-est du champ de blé. En effet, des adultes prélevés sur cette légumineuse puis relâchés au même endroit, aussitôt après avoir été marqués sur le thorax à l'aide d'une peinture, (Enamel, pour modèles réduits) ont été retrouvés, en petit nombre, dans le blé. La deuxième orientation des vols de colonisation (du NO au SE) peut-être rapprochée soit de cultures plus lointaines, soit de la végétation spontanée couvrant les collines qui dominent la plaine 
du Var. Ces coccinelles issues des sites d'hivernation y trouveraient, avant la colonisation du champ de blé, les ressources alimentaires indispensables à leur maturation sexuelle. II est également possible que la haie claire de cyprès qui sépare le blé de la luzerne soit partiellement responsable de la séparation en 2 groupes des adultes colonisateurs.

Comme Ives (1981), nous avons mis en évidence, pour chacune des 3 coccinelles, une relation linéaire entre le nombre de captures sur l'ensemble des pièges englués et le nombre total correspondant d'adultes dans le champ de blé. L'utilisation de cette technique de piégeage pour estimer le niveau des populations dans les cultures paraît difficilement envisageable compte tenu des critiques qui lui ont été faites précédemment (Heathcote, 1978). Au contraire, Ives (1981) pense qu'elle pourrait apporter des informations intéressantes si elle est associée à d'autres méthodes d'appréciation des déplacements.

\section{REMERCIEMENTS}

Nous tenons à remercier R. Boll, E. Franco, L. Giuge, P. Gubanti et J. Molinari pour leur participation à ces travaux.

\section{RÉFÉRENCES}

Ali M.H. \& Azam K.M. (1977) Dispersal of the predatory beetle Coccinella septempunctata L. J. Entomol. Res. 1, 158-193

Andow D.A. \& Risch S.L. (1985) Predation in diversified agrosystems : relations between a coccinellid predator Coleomegilla maculata and its food. J. Appl. Ecol. 22, 357-372

Caprina J.L. \& Walmsley M.R. (1978) Visual responses of some sugarbeet insects to sticky traps and water pan traps of various colors. J. Econ. Entomol. 71, 926927

Davis J.R. \& Kirkland R.L. (1982) Physiological and environmental factors related to the dispersal flight of the convergent ladybeetle, Hippodamia convergens (Guerin-Menaville). J. Kansas Entomol. Soc. 55, 186187

Dean G.J. (1982) Phenology of aphidophagous predators. Ann. Appl. Biol. 101, 182-184
Dowell R. \& Cherry R.H. (1981) Survey traps for parasitoïds and coccinellids predators of the citrus blackfly Aleurocanthus woglumi. Entomol. Exp. Appl. 29, 356-362

Duelli P. (1980) Adaptative dispersal and appetitive flight in green lacewing Chrysopa carnea. Ecol. Entomol. 5, 213-220

Ewert M.A. \& Chiang H.C. (1966) Dispersal of three species of coccinellids in corn fields. Can. Entomol. 98, 991- 001

Heathcote G.D. (1978) Coccinellid beetle on sugar beet in Eastern England. Plant Pathol. 27, 103-108

Hodek I. (1973) Biology of Coccinellidae. Academia, Prague, $266 \mathrm{p}$

Honek A. (1979) Plant density and occurrence of Coccinella septempunctata and Propylea quatuordecimpunctata (Col., Coccinellidae) in cereals. Acta Entomol. Bohemoslov. 76, 308-312

Honek A. (1980) Population density of aphids at the time of settling and ovariole maturation in Coccinella septempunctata L. (Col. Coccinellidae) Entomophaga $25,427-430$

Iperti G. (1965) Contribution à l'étude de la spécificité chez les principales coccinelles aphidiphages des Alpes-Maritimes et des Basses-Alpes. Entomophaga 10, 159-178

Ives P.M. (1981) Estimation of coccinellid numbers and movement in the field. Can. Entomol. 113, 981-997

Kennedy J.S. (1961) A turning point in the study of insect migration. Nature 189, 785-791

Kieckhefer R.W. \& Olson G.A. (1974) Dispersal of marked coccinellids from crops in South Dakota. $J$. Econ. Entomol. 67, 52-54

Kokubu H. \& Duelli P. (1984) Aerial population movement and vertical distribution of aphidophagous insects in cornfields (Chrysopidae, Coccinellidae and Syrphidae) In : Ecology of aphidophaga 2 Symposium (Hodek ed.), Academia, Praha, pp. 279-284

Lapchin L., Ferran A., Iperti G. \& Rabasse J.M. (1987) Coccinellids (Col., Coccinellidae) and Syrphids (Dip., Syrphidae) as predators of aphids in cereal crops : a comparison of sampling methods. Can. Entomol. 119, 815-822

Nakamuta K. \& Saito T. (1985) Recognition of aphid prey by the ladybeetle Coccinella septempunctata bruckü Mulsant (Col. Coccinellidae). Appl. Entomol. Zool. 20, 479-483

Stechmann D.H. (1984) Cereal aphids-aphidophaga associations hedges and fields : can habitat interaction contribute to integrated pest management $I n$ : Ecology of aphidophaga 2 Symposium (Hodek ed.), Academia, Praha, pp. 279-284 